\title{
Leakage of waves from coronal loops by wave tunneling
}

\author{
C. S. Brady, E. Verwichte, and T. D. Arber
}

Physics Department, University of Warwick, Coventry, CV4 7AL, UK

e-mail: chrisb@mcs.st-and.ac.uk

Received 24 August 2005 / Accepted 25 November 2005

\begin{abstract}
To better understand the decay of vertically polarised fast kink modes of coronal loops by the mechanism of wave tunneling, simulations are performed of fast kink modes in straight flux slabs which have Alfvén speed profiles which include a tunneling region. The decay rates are found to be determined by the mode number of the trapped mode and the thickness of the tunneling region. Two analytical models are suggested to explain the observed decay. The first is a extension of the work of Roberts $(1981$, Sol. Phys., 69, 39) to include a finite thickness tunneling region, and the second is a simpler model which yields an analytical solution for the relationship between decay rate, period and the thickness of the tunneling region. The decay rates for these straight slabs are found to be slower than in observations and those found in a previous paper on the the subject by Brady \& Arber $(2005$, A\&A, 438, 733) using curved flux slabs. It is found that the difference between the straight slabs used here and the curved slabs used in Brady \& Arber (2005, A\&A, 438, 733) can be represented as a geometric correction to the decay rate.
\end{abstract}

Key words. Sun: corona - Sun: oscillations - Sun: magnetic fields

\section{Introduction}

The TRACE spacecraft detected spatial displacement oscillations of coronal loops (Aschwanden et al. 1999). The oscillations are a physical displacement of an entire coronal loop. They are normally excited by a local impulsive event such as a flare, and are interpreted as the fundamental standing kink oscillation of the loop. Further work by e.g. Nakariakov et al. (1999) showed that the oscillations were very strongly damped.

The decay of the kink oscillations detected in coronal loops by TRACE (Nakariakov et al. 1999) remains one of the unsolved problems in coronal seismology. The theory of fast magnetoaccoustic waves in magnetic flux tubes shows that they should be trapped by the Alfvén speed minimum at the slab centre (Roberts 1981; Roberts et al. 1983, 1984). Various methods have been suggested to explain the observed decay rates, which are orders of magnitude too fast to be explained by shear viscosity. These methods include resonant absorption (with enhanced shear viscosity) (Nakariakov et al. 1999), resonant mode conversion of quasi-modes (Ruderman \& Roberts 2002), dissipation due to the formation of small scale across the wavefront by Alfvén wave phase mixing (Ofman \& Aschwanden 2002) or coupling to other wavemodes (Roberts 2000). In most of the observations of oscillation coronal loops, the loop is excited by a remote flare, and oscillates out of the loop plane, that is the direction of displacement is perpendicular to the loop plane. In Wang \& Solanki (2004), observations were made of a coronal loop which was oscillating within the loop plane. Oscillations in this manner allow the reduction of the problem into a 2D model, which simplifies the treatment either by numerical means or analytically. In Brady \& Arber (2005, hereafter Paper I), an interpretation of the damping of vertically polarised kink waves in coronal loops was presented based upon the leakage of waves from curved flux tubes due to wave tunneling. There have been other papers published which deal with the effect of curvature on the decay of fast kink modes of magnetic slabs. Selwa et al. (2005) performed similar simulations to those in Paper I, using a different equilibrium magnetic field and density profile. The equilibrium was then perturbed by the production of a shock wave by an initially applied excess of density at the origin of curvature for their loop. This procedure made their model loop oscillate with period and decay time comparable to those observed by Wang \& Solanki (2004). They do not explain the mechanism for the decay of the kink mode excited in their loop, although they do note that their excitation mechanism produces leaky sausage modes, which they claim are responsible for the observed fast waves in their simulations. Their model does yield a tunneling region similar to that in Paper I and although no simulations have been performed to test this hypothesis, their results seem to be consistent with those presented in Paper I. Another, earlier paper which attempts to include curvature effects is Van Doorsselaere et al. (2004), who considered the modification of the quasimodes of a loop structure due to an induced curvature. They find enhanced decay rates for the quasi-modes compared with a straight loop model. Here, the decay is due to resonant coupling between the global kink oscillation and a local torsional Alfvén mode. They find that curvature has only a small effect on the decay rate of modes in the loops, but a significant difference between the model used in that paper and that used in 


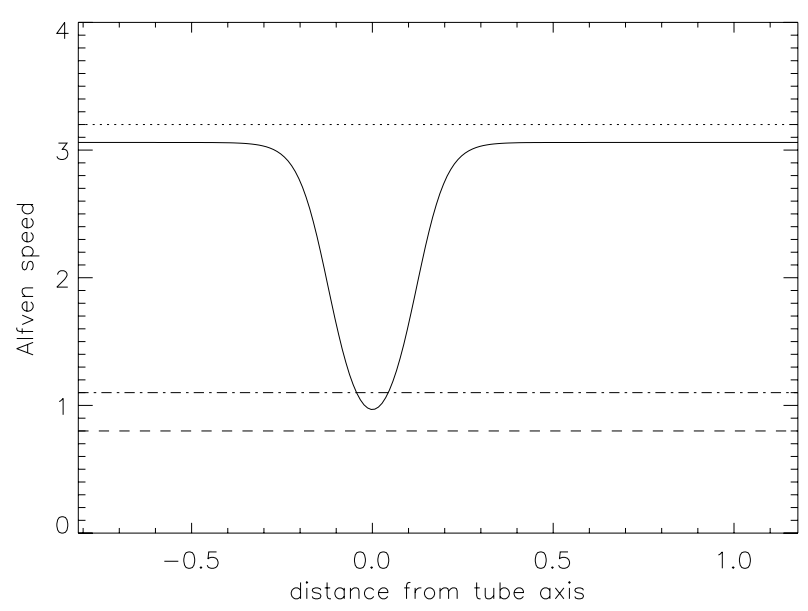

Fig. 1. Alfvén speed vs. distance from slab axis for a slab without a tunneling region. The dot-dashed line represents a mode that is trapped in the slab in this system. The dotted line represents a mode that would be travelling both inside and outside the slab, and the dashed line represents a mode which would be evanescent everywhere. This figure was included in Paper I, where it was used simply to demonstrate the concept associated with wave leakage, and this model is not strictly applicable to the curved slab model used in that paper. It is the correct model for the straight slab considered in this paper.

Paper I is that they considered a linear Alfvén speed profile (except for a thin layer at the loop edge). This type of model does not include the tunneling region which is essential to the results presented in Paper I (see also Verwichte et al. 2006). In Paper I, simulations were performed with a simple model magnetic slab which had been given a finite radius of curvature.

It was found that placing a simple equilibrium magnetic slab model in curved geometry leads to the modification of the Alfvén speed profile, so that there is a finite thickness tunneling region through which a wave may tunnel and escape from the slab as fast waves. For a kink mode with a given frequency, $\omega$, and longitudinal component of the wavevector, $k$, the requirement for it to be locally propagating is that

$\frac{\omega}{k}>v_{\mathrm{A}}^{2}$

where $v_{\mathrm{A}}$ is the local Alfvén speed.

In Fig. 1 the Alfvén speed profile of a simple straight slab is shown. For a straight magnetic slab, the mode can be represented by its longitudinal phase speed $C_{\mathrm{ph}}=\omega / k=A$ where $A$ is a constant, and three possible values of $A$ are plotted in Fig. 1. The dotted line represents a mode where Eq. (1) is satisfied everywhere. This represents a mode which has wavelike structure everywhere and is not confined in the slab. The dashed line has a value of $A$ such that Eq. (1) is never satisfied, and represents a solution which is evanescent everywhere and is obviously unphysical. The dot-dashed line represents a mode where Eq. (1) is satisfied inside the slab but not in the external regions. The mode would therefore be confined to the slab with only evanescent tails in the external regions.

When developing a model of a curved flux slab, it was decided that the simplest model was to curve a simple magnetic slab so that the central axis of the slab was now an arc of a circle, and then to develop a magnetic field profile which has field

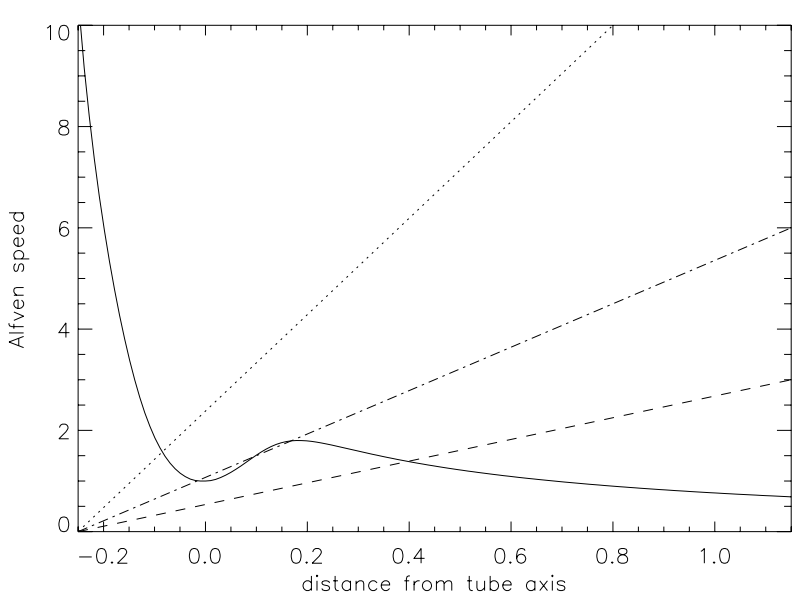

Fig. 2. Alfvén speed vs. distance from slab axis for slab with a tunneling region with lines representing different modes in a curved geometry. The dot-dashed line represents a mode that would be trapped in a slab without a tunneling region, but can leak through tunneling in this system. The dotted line represents a mode that would be travelling both inside and outside the slab, and the dashed line represents a value of $\omega / k$ which cannot exist, since it would be evanescent everywhere.

lines which are coaxial with the flux slab axis. The simplest magnetic field model which has semicircular field lines is the field surrounding an infinitely long straight wire. This model changes the Alfvén speed profile to that shown in Fig. 2. It also changes the longitudinal phase speed to be $C_{\mathrm{ph}}=\omega / k=A r / r_{0}$, where $A$ is still a constant, $r$ is the radial coordinate with an origin at the centre of curvature of the loop and $r_{0}$ is a reference radius. The reason for this modification can be seen at follows. The mode number, $m$, is defined as

$m=\frac{L k}{\pi}$

where the arclength of a semicircular arc is given by $L=\pi r$. For $m$ to be constant for all radii, $k$ needs to be of the form

$k(r)=\frac{m}{r}$.

It should be noted that this functional form is only valid if the field lines are assumed to be semicircular, and a more realistic field model would produce a different form. However, if the wave is forced to follow any arbitrary curved line, the same principle will locally apply everywhere, with $r$ now representing the distance of the local radius of curvature at any point on the curve. The effect given here is therefore a purely geometric one, and unconnected with the choice of model.

The same numerical values for $A$ as were used to derive Fig. 1 are substituted into the revised equation for the phase speed and plotted again. There are some interesting changes to what they represent. For all values of $A$, there are now regions where the mode satisfies Eq. (1), and regions where is does not. For the dotted line in Fig. 2, the mode will still be propagating both inside the slab, and at all radii greater than that of the slab axis, but there is a finite minimum radius below which this mode will not propagate. Similarly, the dashed line represents a mode which is still not propagating in the slab, but now becomes propagating at a finite distance from the slab 


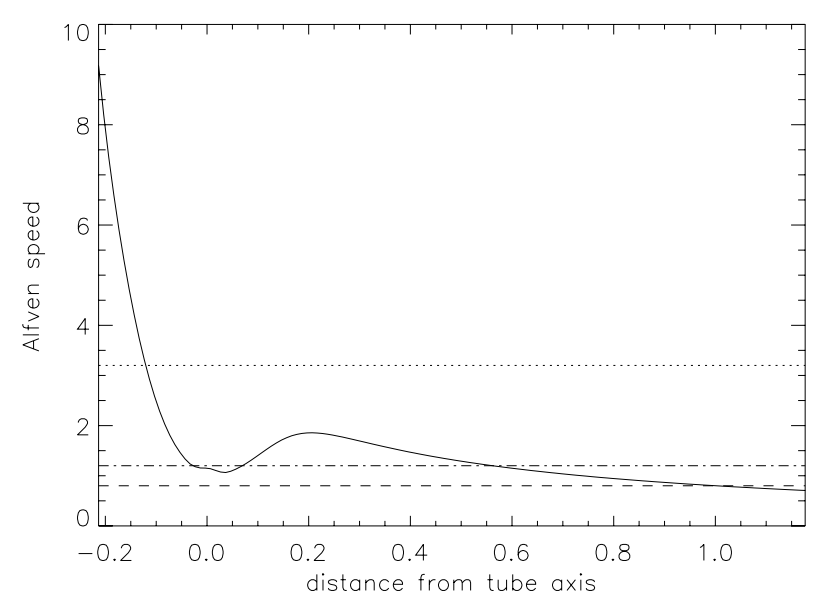

Fig. 3. Alfvén speed vs. distance from slab axis for slab with a tunneling region with lines representing different modes in a straight slab geometry. The dot-dashed line represents a mode that would be trapped in a slab without a tunneling region, but would can leak through tunneling in this system. The dotted line represents a mode that would be travelling both inside and outside the slab, and the dashed line represents a value of $\omega / k$ which cannot exist, since it would be evanescent everywhere.

axis, although it is likely that this will not significantly affect the behaviour of this mode. The case of the dot-dashed line is the most interesting. Here, the mode is propagating in the slab, evanescent in the region just outside the slab, and propagating again outside this region. This is a problem very similar to that of quantum tunneling and in wave tunneling in classical optics and acoustics. Simulations were performed in Paper I to evaluate the efficiency of the leakage of waves from curved slabs with the profile given in Fig. 2, and it was found that low harmonic standing kink modes decayed on timescales even more rapid than shown by observations.

If leakage is due to wave tunneling, then an increase in the thickness of the tunneling region would be expected to lead to a decrease in the decay rate, but the exact effect of changing the thickness is not immediately clear. Therefore, the purpose of this paper is to better quantify the effect of leakage due to tunneling by determining how a wider range of parameters affect decay rates and to separate the effects of tunneling from curvature effects.

If the plot in Fig. 2 is changed to show the effect of combining an Alfvén speed profile like that for the model curved slab with the $\omega / k$ profiles for a straight slab, then certain features can be observed (see Fig. 3). Details on how this model is created are given in the next section. Firstly, the lines still correspond to modes which have the same behaviour, the dotted line is still propagating both inside and outside the slab, the dashed line still represents a mode which will be decaying inside the slab, but will be oscillatory at some finite radius, and the dotdashed line still represents a mode that can propagate inside the slab, but can only leave it by tunneling. However, in Fig. 2, the dot-dashed line just grazes the top of the tunneling region, having to tunnel through only a thin region, whereas in Fig. 3 it has to tunnel through a longer region, in fact some 10 times longer. This shows that if the theory that the decay is caused by wave tunneling is correct then the decay times of straight slabs, even with the same Alfvén speed profile, will be significantly longer than those for curved slabs.

This paper is structured as follows. In Sect. 2, the model used in the numerical simulations is presented, and the solver used in the simulations is introduced. In Sect. 3, the results from the numerical model are described. In Sect. 4, the analytical models are introduced, and some results from them are presented. In Sect. 5, the conclusions are presented and the numerical and analytical models are compared.

\section{Numerical model}

All simulations are performed using a 2D Lagrangian remap code detailed in Arber et al. (2001). The simulations are performed on a $300 \times 200$ Eulerian grid, and the results are tested for convergence by doubling the resolution $(600 \times 400)$. All results tested varied by less than $1 \%$ when the resolution was doubled.

The MHD equations solved are

$\frac{\partial \rho}{\partial t}+\nabla \cdot(\rho \boldsymbol{V})=0$

$\frac{\mathrm{d}}{\mathrm{d} t}\left(\frac{P}{\rho^{\gamma}}\right)=0$

$\rho \frac{\mathrm{d} \boldsymbol{V}}{\mathrm{d} t}=-\nabla P-\frac{\boldsymbol{B}}{\mu_{0}} \times(\nabla \times \boldsymbol{B})+\nabla \cdot \boldsymbol{S}$,

$\frac{\partial \boldsymbol{B}}{\partial t}=\nabla \times(\boldsymbol{V} \times \boldsymbol{B})$

where $S$ is the stress tensor whose components are $S_{i j}=$ $v\left(\epsilon_{i j}-\frac{1}{3} \delta_{i j} \nabla \cdot \boldsymbol{V}\right), \epsilon_{i j}=\frac{1}{2}\left(\frac{\partial V_{i}}{\partial x_{j}}+\frac{\partial V_{j}}{\partial x_{i}}\right), \delta_{i j}$ is the Kronecker delta function and $v$ is the viscosity. The basic quantities used in the simulation are the density $\rho$, the pressure $P$, the magnetic field vector $\boldsymbol{B}$ and the velocity vector $\boldsymbol{V}$.

The code runs in dimensionless units, chosen such that the equilibrium magnetic field $\left(B_{0}\right)$, density $\left(\rho_{0}\right)$ and Alfvén speed $\left(C_{\mathrm{A} 0}\right)$ at the slab centre are all unity. In Paper I, lengths were normalised to the major radius of the loop. Here the normalisation is chosen so that the loop length remains the same as in Paper I. This means that the loop footpoints are at $\pm \pi / 2 . L_{0}$ is the basic unit of length, and is chosen so that the loop length is $\pi$. Time is again normalised in units of $L_{0} / C_{\mathrm{A} 0}$, where $C_{\mathrm{A} 0}$ is the Alfvén speed at the loop centre. Also, in these units, $\mu_{0}=1$. A straight equilibrium flux slab was introduced parallel to the $z$ axis, with an Alfvén speed profile which includes a tunneling region. The simulation takes place in the $x-z$ plane, with a constant equilibrium magnetic field parallel to the $z$ axis, i.e. $B_{0 z}=B_{0}, B_{0 x}=0$. This field was chosen as it is the simplest potential field which is everywhere coaxial with the slab.

In Paper I the Alfvén speed profile in normalised units was given by

$C_{\mathrm{A}}^{2}=\frac{B_{0}^{2}}{\rho_{0}}=\frac{B_{0}^{2}\left(\frac{r}{r_{0}}\right)^{-2}}{\left(\left(\rho_{\max }-\rho_{\infty}\right) \operatorname{sech}^{2}\left(\frac{r-r_{0}}{w}\right)+\rho_{\infty}\right)}$, 


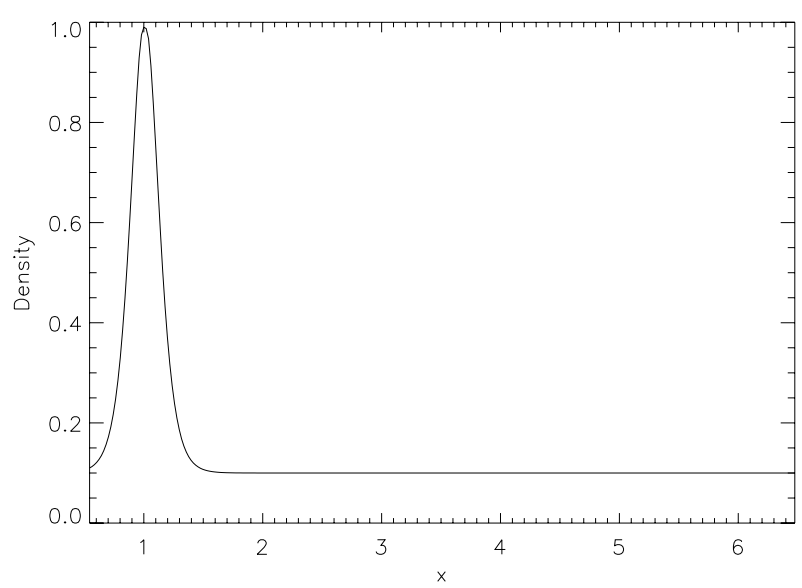

Fig. 4. The density profile for a straight slab with $n=0.0$. This is a simple straight flux slab profile with no tunneling region. The $x$-axis does not start from 0 since it is known from preliminary simulations and results obtained in Paper I that waves are not transmitted through the inner edge of the slab $(x \rightarrow 0)$. Therefore, to decrease runtime, the domain was not simulated to include $x=0$. In order to be comparable with Paper I, it was necessary that the slab centre be at $x=1$.

where $r_{0}$ was the radius of the slab axis, $\rho_{\max }$ was the density at the slab center, $\rho_{\infty}$ is the density at $r=\infty$ and $w$ is the slab width, where $B_{0}$ is the values of the magnetic field at $r=r_{0}$, i.e. $B_{0}=1$ in normalised units.

This profile follows from the choice of a symmetric Epstein density profile, combined with a magnetic field that drops off as $1 / r$. Converting this profile to Cartesian coordinates is trivially simple, requiring only the exchange of the radial coordinate $r$ with $x$, the Cartesian coordinate normal to the slab axis. In order to study the effect of varying the tunneling thickness, a more general density profile is therefore chosen. The corresponding Alfvén speed profile is therefore

$$
C_{\mathrm{A}}^{2}=\frac{B_{0}^{2}\left(\frac{x}{x_{0}}\right)^{-n}}{\left.\left(\left(\rho_{\text {slab }}-\rho_{\text {extern }}\right) \operatorname{sech}^{2}\left(\frac{x-x_{0}}{w}\right)+\rho_{\text {extern }}\right)\right)},
$$

where $x_{0}$ is the $x$ position of the slab axis (here chosen to be 1 after normalisation), $\rho_{\text {slab }}=1$ was the density at the slab center and $w$ is the slab width (equal to 0.25 for all the simulations performed here). The power index $n$ controls the overall shape of the density profile. $B_{0 z}$ is now the constant value of the background magnetic field, and is still 1 due to normalisation. Given the magnetic field $B_{z}=1$ everywhere, the density profiles obtained from Eq. (9) are no longer symmetric Epstein profiles. In fact, they only tend to a finite value for $x \rightarrow \infty$ in the $n=0$ case, but rather tend to 0 for $n>0$ or tend to $\infty$ for $n<0$. Therefore, $\rho_{\text {extern }}$ is not the density for $x \rightarrow \infty$ except in the $n=0$ case. Two examples of this are given in Figs. 4 and 5, for $n=0$ and $n=2$ cases, respectively. This means that the density contrast ratio $\rho_{\text {slab }} / \rho_{\text {extern }}$ is not as well defined as it is in Paper I. The slab created by this model has a characteristic tunneling thickness, $T$, which is defined as the distance between the points where the mode becomes non-propagating until it becomes propagating again. It can be shown that the power index $n$ controls $T$ from $T=\infty$ for $n=0$ to $T=0$ for $n=\infty$. Therefore, we will call $n$ the tunneling parameter. In the

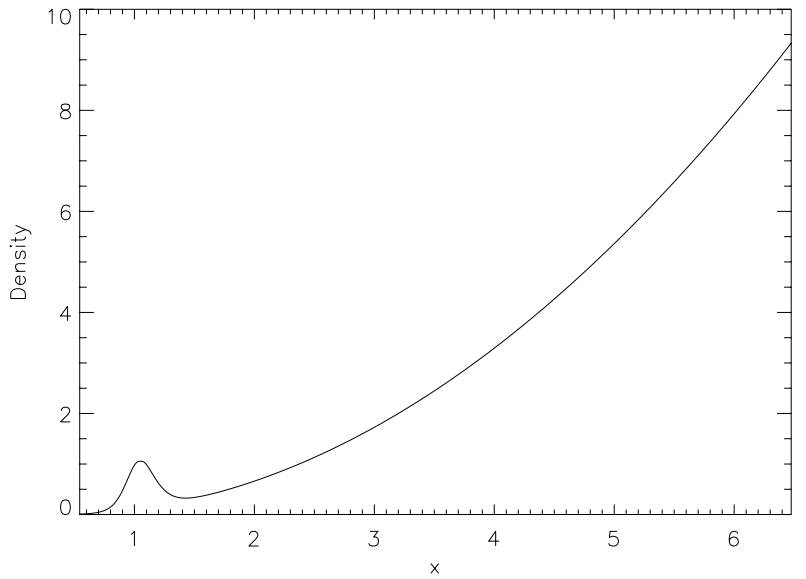

Fig. 5. The density profile for a straight slab with $n=2.0$. This is a straight flux slab with an Alfvén speed profile identical to the radial Alfvén speed profile in Paper I.

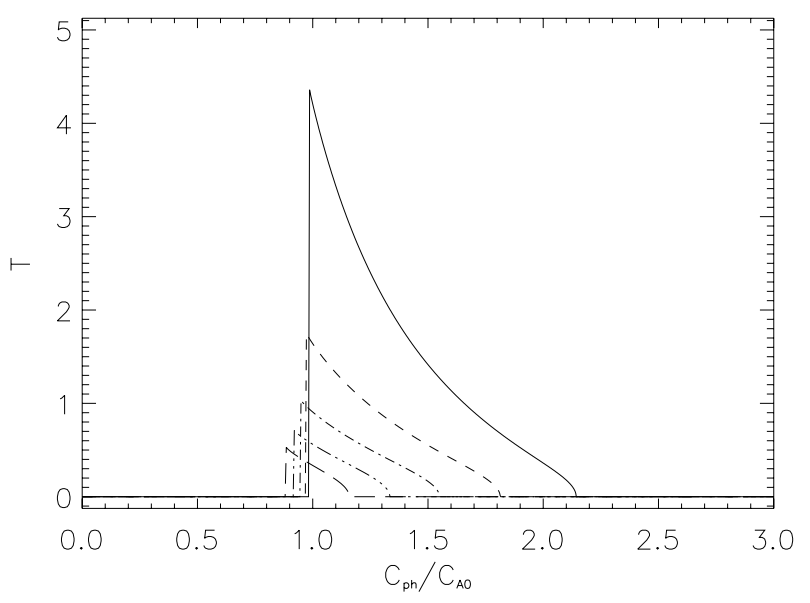

Fig. 6. The thickness of the tunneling region for different values of $n$ with a density contrast ratio of 10 , and a slab aspect ratio of 2.0. (The different lines refer to different values of $n$.) The solid line refers to $n=1$, the dashed line to $n=1.5$, the dot-dashed line to $n=2.0$ etc., with a separation of 0.5 between all the lines.

case of $n=2$, the Alfvén speed profile from Paper I is recovered. Note that $n$ is not required to be an integer, and the value of $T$ also depends on the phase speed of the wavemode. The equation relating the thickness of the tunneling region to the tunneling parameter is transcendental, but the tunneling thickness can be determined numerically in terms of the ratio of the phase speed $\left(C_{\mathrm{ph}} / C_{\mathrm{A} 0}\right)$ of the mode in the slab to the Alfvén speed at $x=x_{0}$. This relation can be seen for a variety of values of $n$ in Fig. 6. There are two notable features here. Firstly, for sufficiently small values of $C_{\mathrm{ph}} / C_{\mathrm{A} 0}$ the tunneling thickness is not a well defined quantity. This corresponds to the wave mode not being propagating even in the slab, and so is not surprising. For large $n$, modes exist for $C_{\mathrm{ph}} / C_{\mathrm{A} 0}<1$, which would appear to suggest that modes can propagate which do not satisfy Eq. (1) even at the slab axis. This is not as significant as it first seems, since for large $n$, the minimum in the Alfvén speed is not at $x=x_{0}$, but at some smaller value of $x$ in the slab, whereas $C_{\mathrm{A} 0}$ is still defined as the Alfvén speed at $x=x_{0}$. 
The only remaining feature of interest is that the thickness of the tunneling region decreases significantly if the phase speed of the mode in the slab is significantly higher than the Alfvén speed at the slab axis.

Having specified a magnetic field profile and the density, the temperature is then chosen so that the model is in hydrostatic equilibrium under the assumption of an ideal gas with a polytropic index of $5 / 3$. The plasma- $\beta$, defined as the ratio of the gas pressure to the magnetic pressure, is constant to machine precision at equilibrium with a value of 0.1 .

To allow the waves to leave the numerical domain, a damping region is introduced in which the velocity is artificially decreased away from the slab. In this region, the velocities returned by the core solver are multiplied by a value less than one, with the value smoothly varying from 1 to 0 over the damping region. If the damping region is long compared with the wavelength of the radiated fast waves then reflection from the damping region is negligible and the boundary can be considered effectively open. All other boundaries are line-tied, except for the $z=-\pi / 2$ boundary, where kink oscillations are introduced into the slab by boundary driving, with the velocity perpendicular to the slab axis specified at the centre of the slab $(x=1)$ at $z=-\pi / 2$. The function used is the same as in Paper I

$V_{x}=V_{0} \mathrm{e}^{-\frac{\left(x-x_{0}\right)^{2}}{\sigma^{2}}} \sin \omega t$

where $x_{0}=1$ is the $x$ position of the flux slabs central axis, $\sigma$ is the width of the driver, which is smaller than, but comparable to the slab width (specifically $\sigma=0.2$ ) and $\omega$ is the driving frequency and is in the range 6.18 to 9.33 , this range being chosen to explore the full range of wavelengths unimpeded by either viscous dissipation or mode distortion by the presence of an antinode at one footpoint due to the driver. The amplitude of the driving, $V_{0}$, is the same for all models and was chosen so that the maximum value of the driving function is Alfvén Mach 0.1, i.e. $V_{0}=0.1$ in units normalised to the Alfvén speed in the slab. The system is driven until a wave is well established in the slab. The driver is then ramped down until the lower boundary becomes line-tied. The system is then allowed to equilibrate until it is in one dominant eigenmode. Due to this driving, the slab will be in a superposition of several eigenmodes, but simulations confirm that it has a preferred mode, representing the eigenmode with the frequency nearest the driving frequency, in which more than $95 \%$ of the kinetic energy in the slab resides. Once the dominant preferred mode is established, its decay rate is measured.

In observations of kink oscillations in coronal loops, the oscillations are excited by nearby impulsive activity (Aschwanden et al. 1999), not by footpoint driving, but the purpose of this paper is to understand in more detail the tunneling of fast kink modes of flux slabs once excited, not the excitation mechanism itself, and therefore the more controllable method of footpoint driving is preferable. A uniform tensor viscosity is applied in this paper to suppress the formation of grid-scale noise. The normalised viscosity used has a value of $v=2 \times 10^{-5}$.

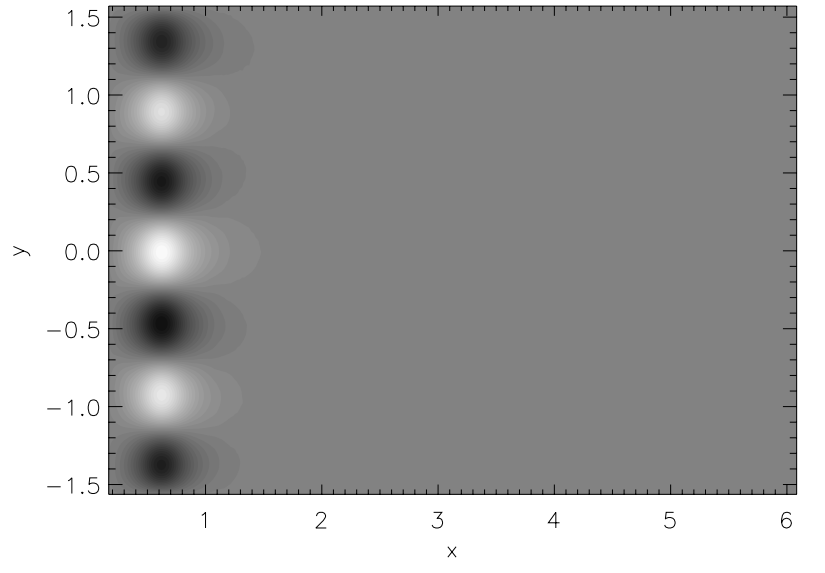

Fig. 7. $V_{\text {perp }}$ for $n=0$ (i.e. no tunneling region). The wave is well confined to the body of the slab.

The parameter $\psi$ is introduced as

$\psi=\int\left|V_{\text {perp }}\right| \mathrm{d} \boldsymbol{x}$,

where the integral is over the slab width, i.e. a distance of $w$ on either side of the slab axis, and $V_{\text {perp }}$ is the velocity perpendicular to the local magnetic field. Here $|A|$ is used to mean the absolute value of $A$. This diagnostic was introduced in Paper I, where it was compared with the diagnostic used in observations. The diagnostic commonly used in observations is simply to plot the displacement of the loop apex and fit a damped sine wave to the resulting points. Although it is obvious that the decay of the velocity of the loop top will be the same as the decay of the amplitude of the displacement of the loop maximum, in Paper I the two diagnostic methods were tested against each other and found to agree in both period and damping time to within error. The use of $\psi$ as a diagnostic is retained here since the calculation of the position of the loop maximum requires further calculations and increases error. Also, although the displacement of the loop top is a good diagnostic for the primary mode of oscillation of a loop, it is a poor diagnostic for higher modes.

The quantities varied in this paper are the driver frequency $\omega$, and the tunneling parameter $n . \omega$ is varied to test whether the same or similar results are obtained for a straight slab as for a curved slab with the same Alfvén speed profile, and $n$ is varied to test how the decay of a particular wave mode is changed by changing the thickness of the tunneling region.

\section{Numerical results}

To confirm that the straight slab without a tunneling region does not leak waves, simulations were initially performed with $n=0$. These simulations give a velocity profile as in Fig. 7.

The waves are confined in the body of the slab, but there is still a finite decay time due to the presence of the tensor viscosity. The decay time observed for this simulation matches exactly that predicted by the values of $v$ introduced into the code. 


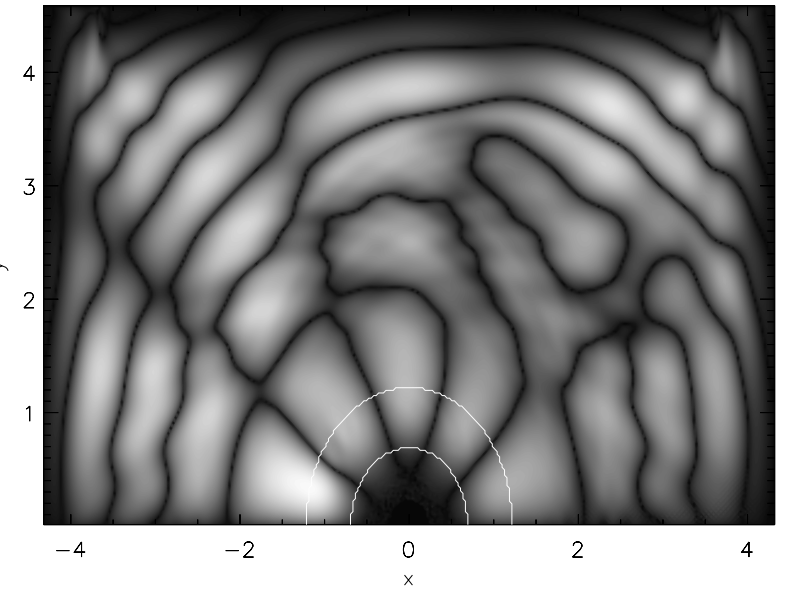

Fig. 8. $\left|V_{\text {perp }}\right|^{0.5}$ for the 5 th harmonic eigenmode for a curved slab. The solid white lines represent the region which is one slab half-width on either side of the slab centreline.

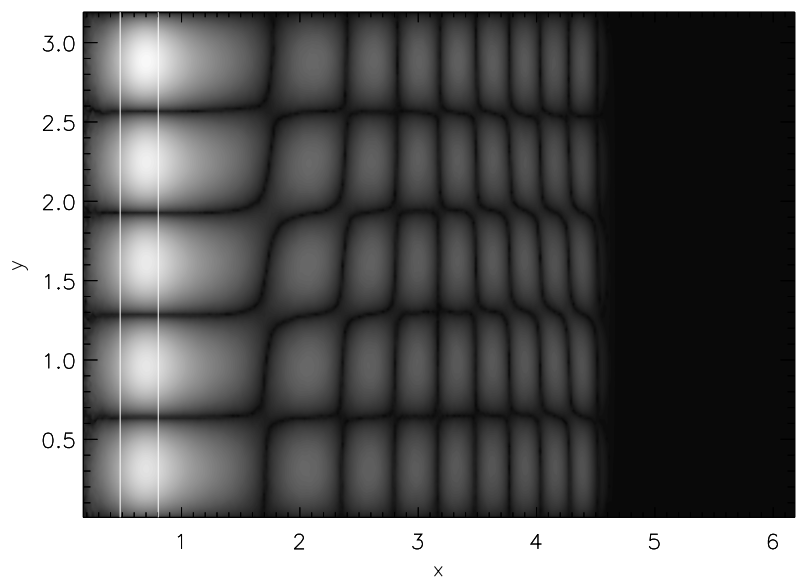

Fig. 9. $\left|V_{\text {perp }}\right|^{0.5}$ for the 5 th harmonic eigenmode for a straight slab. The solid white lines represent the region which is one slab half-width on either side of the slab centreline. Although it is not clear in this diagram, the amplitude of the waves decreases rapidly away from the tube due to the previously mentioned increasing density. The apparent disappearance of the radiated waves at $x=4.5$ is in fact due to the necessity to choose contours which allow as much as possible of the wave pattern to be seen clearly.

Simulations are then performed to check that the conversion to straight slab geometry has not stopped the leakage of the wavemode. A simulation is performed of a straight slab with the exactly equivalent Alfvén speed profile to the curved slab $(n=2)$, with a driver frequency $(\omega)$ which should excite the fifth harmonic, the longest wavelength simulated in Paper I. In fact, the simulation stimulated the 4th harmonic in the straight slab. This was then scaled down until the 5 th harmonic was successfully excited. The change in driver period required was only small (about 5\%), and the most likely cause of this change is some small change due to curvature. By inspection of Figs. 8 and 9, it is clear that the slab still radiates fast waves. Figure 10 shows that the decay rate of the straight slab is lower than that of the curved slabs, with only about an order of magnitude difference between the decay rates due to viscosity and the decay rates due to tunneling loss. As in Paper I, the curves show



Fig. 10. The time series from a simulation for the seventh harmonic for the straight slab model (dotted line) and the curved slab model (solid line). The higher decay rate after the initial transient period in the straight slab case is clear. It is clear that the straight tube model noticeably has two different decay regimes, the first with a decay constant of $0.029 \pm 0.001$, and the second with a decay constant of $0.0015 \pm 0.0003$. More detailed observations of the two regimes shows that the first section is dominated by strongly asymmetric loss at the driven footpoint, and is assumed to be related to the driving procedure. The second phase is marked by essentially uniform decay, and represents the decay of the eigenmode of the slab.

oscillations with two periods, a short period which corresponds to the period of the wavemode in the slab, and a longer period which is caused by an Alfvén pulse generated by the driver switch-off. Decreasing the driver over a longer period reduces the amplitude of this mode, but increases runtime significantly and does not change the value of the obtained decay rate. By comparing the results for a simulation with no tunneling region $(n=0)$ and for a relatively thin tunneling region $(n=3)$, it is hoped that there will be an obvious difference in the observed decay rates. In Fig. 11, this is shown to be true, with the decay for the case $n=0$ being entirely consitent with the expected viscous decay rate, and the decay for $n=3$ significantly faster.

The tunneling parameter, $n$, and the driver period are then systematically varied to obtain the functional dependence of the decay constant on the thickness of the tunneling region, and on the period of the driver, and hence the period of the standing mode. The results for the seventh harmonic shows that there is a logarithmic relationship between the decay time and the tunneling region thickness until the decay rate becomes dominated by the viscous damping of waves when the thickness of the tunneling region, $T=1.35$, i.e. 5.4 times the slab half width (see Fig. 12). The picture is similar for other modes (Fig. 13). Since viscous damping scales as $k^{2}$, waves with shorter wavelength actually have shorter decay times, for thick tunneling regions, than waves with longer wavelengths. This can be seen trivially by considering the form of the viscous operator. The simplest viscous operator has the form $\mu \nabla^{2} v$, and if this operator is applied to a wave solution of the form $\mathrm{e}^{\mathrm{i} k x-\mathrm{i} \omega t}$, then the effect of viscosity scales as $k^{2}$. If simulations are performed for $n=0$ for all wavelengths, and these are assumed to be the "baseline" decay rates, then the damping rate due to tunneling still increases with increasing wavelength. This can be tested 


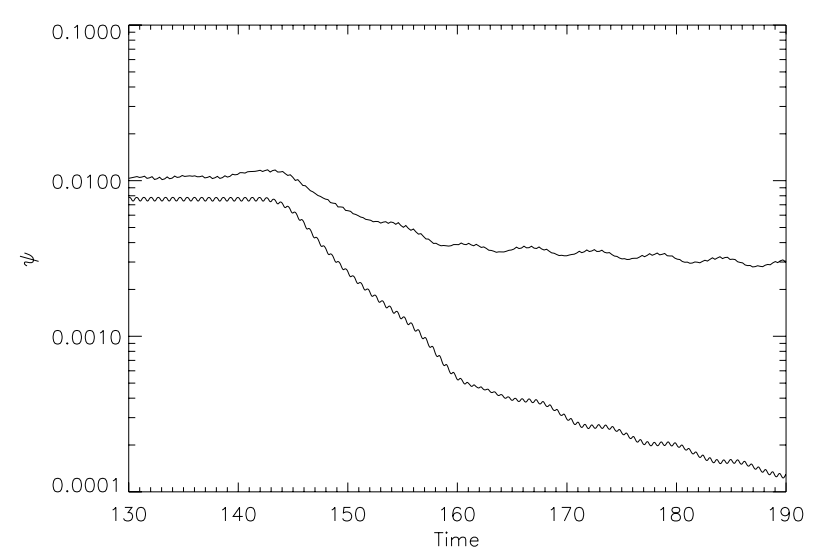

Fig. 11. The time series for two simulations of the seventh harmonic. The upper line is for $n=0.0$, and shows a slow decay characterised by viscous dissipation. The lower line is for $n=3.0$ and shows a more rapid decay which can be seen in contours of $V_{\text {perp }}$ to be characterised by wave leakage.

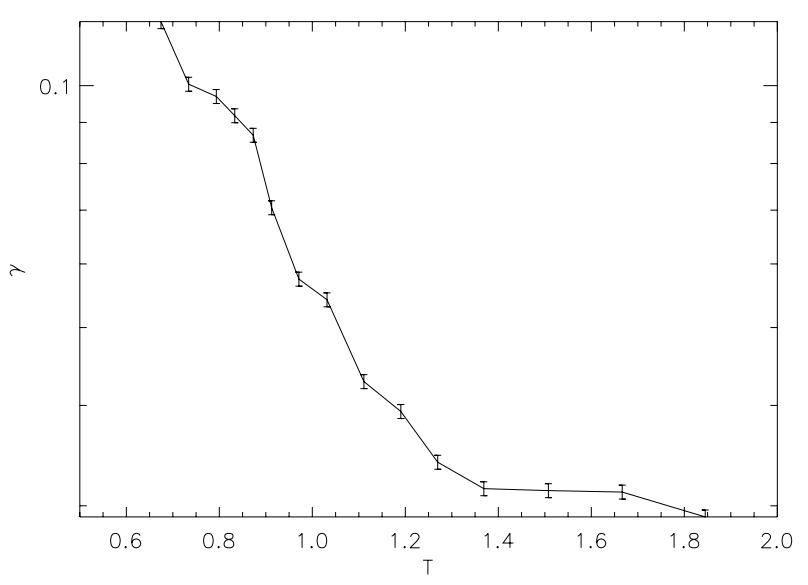

Fig. 12. Decay rate versus tunneling region thickness for the 7th harmonic. The decay time is $15.4 \pm 0.1$ times the period at minimum. The constant high decay time for thick tunneling regions is consistent with the decay time due to viscous damping with the value of viscosity used in this code.

by increasing the resolution of the simulation, which lowers the real-unit viscosity. These results take many times longer to run than the previous simulations, and so were not used for the production of the results presented in this paper. Since the viscosity is several orders of magnitude larger than would be expected to be present in the corona, it is reasonable to ignore any contribution to the damping due to viscosity. Simulations with lower viscosity show that this approach is valid, with the damping rate for a slab with no tunneling region $(n=0)$ tending to zero in the limit of zero viscosity, but reducing viscosity requires higher resolutions, increasing runtimes unacceptably.

\section{Theory}

In order to better understand the results of these simulations, two simple analytical models were derived. The first one is a modification of the model of the oscillation modes of a straight slab derived by Roberts (1981) to include a tunneling region. In that paper, a magnetic slab was modelled as three regions

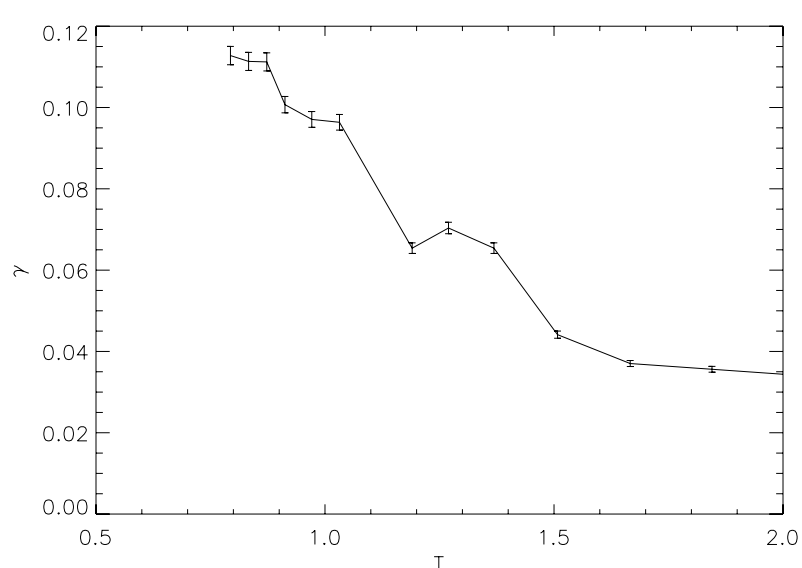

Fig. 13. Decay rate versus tunneling region thickness for the 5 th harmonic. Again, for long tunneling distances, the decay is dominated by viscous dissipation.

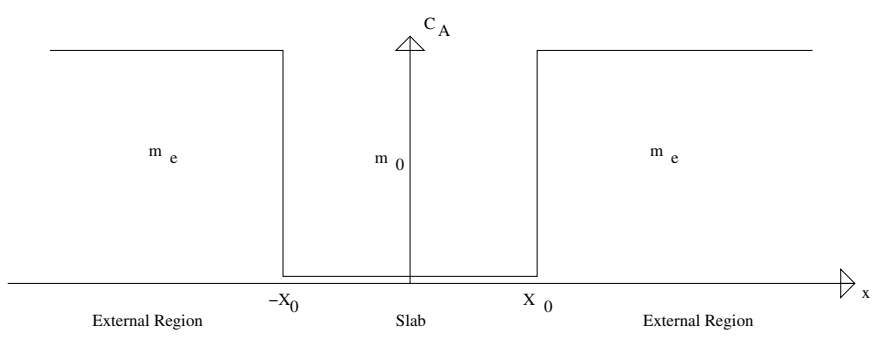

Fig. 14. The slab model in Roberts (1981). Attention was restricted to modes where the solutions in the two external $\left(m_{\mathrm{e}}\right)$ regions were evanescent.

(see Fig. 14). There are two external regions where there is no magnetic field, and density $\rho_{\mathrm{e}}$ and an inner region with a magnetic field that is parallel to the slab axis, and density $\rho_{0}$. The solutions in the external regions, which are here used to refer to the two infinite thickness barriers on either side of the slab are assumed to be evanescent.

In Roberts (1981), the linearised MHD equations were rearranged to yield an equation for the perturbations in the velocity component across the slab

$\frac{\mathrm{d}^{2} v_{x 1}}{\mathrm{~d} x^{2}}-m_{\alpha}^{2} v_{x 1}=0$

where $v_{x_{1}}$ is the perturbation in the velocity component across the slab and $m_{\alpha}$ is either $m_{\mathrm{e}}$ outside the slab or $m_{0}$ inside the slab. In Roberts (1981), $m_{\alpha}$ was calculated for a finite plasma- $\beta$. Since our simulations take place with a small but finite plasma- $\beta$, the problem is simplified by using the zero plasma- $\beta$ approximation. $m_{\alpha}$ therefore becomes

$m_{\alpha}^{2}=k_{z}^{2}-\omega^{2} / C_{\mathrm{A} \alpha}^{2}$,

where $C_{\mathrm{A} \alpha}$ is the Alfvén speed in either the external $\left(C_{\mathrm{Ae}}\right)$ or internal $\left(C_{\mathrm{A} 0}\right)$ regions.

The general solution of Eq. (12) is of the form $v_{x 1}=$ $A_{\alpha} \mathrm{e}^{m_{\alpha} x}+B_{\alpha} \mathrm{e}^{-m_{\alpha} x}$ with $m_{\alpha}$ the root of $m_{\alpha}^{2}$ with a positive real part, and $A_{\alpha}$ and $B_{\alpha}$ integration constants in medium $\alpha$. The basic equation remains valid for the slab with a tunneling region, so this model can easily be changed to include a tunneling region by changing one of the external regions to be of a finite 


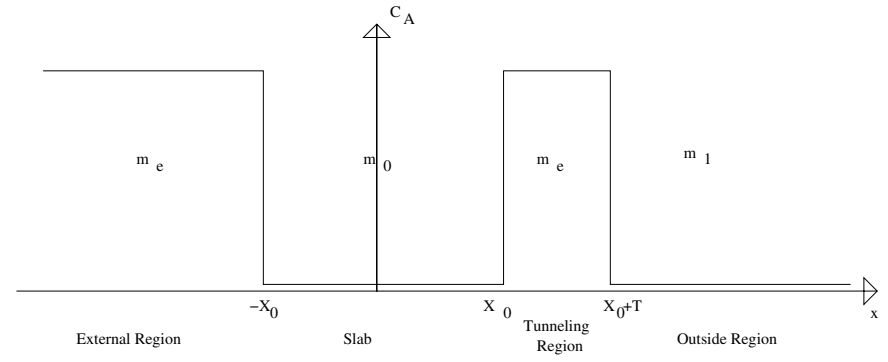

Fig. 15. The modified slab model from Roberts (1981). Again, solutions in the two regions labelled $m_{\mathrm{e}}$ are restricted to being evanescent, and the solution in the region $m_{1}$ is restricted to being an outward propagating wave.

thickness, and introducing a region outside of that barrier (see Fig. 15). In this more complex system, the nomenclature has to be slightly changed. The region of enhanced density will still be called the inner region, or the slab, but the term "external region" will now only be used to refer to the infinite thickness barrier at $x<-x_{0}$. The finite thickness barrier will now be defined as the tunneling region $\left(x_{0}<x<x_{0}+T\right)$, and the area outside the finite thickness barrier will be called the external region $\left(x>\left(x_{0}+T\right)\right)$. The solution in the external region, denoted with subscript " 1 ", is forced to be an outward propagating wave, i.e. the integration constants are chosen such as to retain the solution to Eq. (13) with the imaginary part of $m_{1}$ positive. This reflects the solution to Eq. (13) that propagates in the positive $x$-direction. The system of equations are now solved for the integration constants, using the same technique as in Roberts (1981). This yields

$$
\begin{gathered}
\left(m_{\mathrm{e}}-m_{1}\right)\left[\left(m_{\mathrm{e}}-m_{0}\right)^{2}-\left(m_{\mathrm{e}}+m_{0}\right)^{2} \mathrm{e}^{4 m_{0} x_{0}}\right]= \\
\left(m_{\mathrm{e}}+m_{1}\right)\left(m_{\mathrm{e}}^{2}-m_{0}^{2}\right)\left(1-\mathrm{e}^{4 m_{0} x_{0}}\right) \mathrm{e}^{-2 m_{\mathrm{e}} T},
\end{gathered}
$$

where $m_{0}$ and $m_{\mathrm{e}}$ are the roots with positive real parts, and $m_{1}$ is the root with a positive imaginary part reflecting wave propagating in the positive $x$-direction in the external region. In the limit $m_{\mathrm{e}} T \rightarrow \infty$, the right-hand-side of Eq. (14) tends to zero and the dispersion relation of Roberts (1981) is recovered:

$\left(m_{\mathrm{e}}-m_{0}\right)^{2}=\left(m_{\mathrm{e}}+m_{0}\right)^{2} \mathrm{e}^{4 m_{0} x_{0}}$.

In this limit, for trapped modes $\left(C_{\mathrm{A} 0}<C_{\mathrm{ph}}<C_{\mathrm{Ae}}\right), m_{\mathrm{e}}$ is real and $m_{0}$ is purely imaginary.

In order to gain insight into the influence of $T$, we examine the case of $m_{\mathrm{e}} T$ finite but large, such that $\mathrm{e}^{-2 m_{\mathrm{e}} T} \sim O(\epsilon)$, with $0<\epsilon \ll 1$. The mode frequency may then be written as $\omega=\bar{\omega}-\epsilon \gamma$, where $\bar{\omega}$ is a solution of Eq. (15) and $\gamma$ is a small perturbation of $O(\epsilon)$. The dispersion relation (14) is then linearised and an expression for $\gamma$ is derived:

$\gamma=\Upsilon \frac{\bar{m}_{\mathrm{e}} \bar{m}_{0}}{k_{\mathrm{z}}^{2}}\left(\frac{C_{\mathrm{A} 0}}{C_{\mathrm{ph}}}\right)^{2} \bar{\omega} \mathrm{e}^{-2 m_{\mathrm{e}} T}$,

where

$\Upsilon=\frac{1}{2} \frac{\left(\bar{m}_{\mathrm{e}}+\bar{m}_{1}\right)}{\left(\bar{m}_{\mathrm{e}}-\bar{m}_{1}\right)} \frac{E}{F+G+H}$, and

$$
\begin{aligned}
E & =\left(\bar{m}_{\mathrm{e}}+\bar{m}_{0}\right)\left(\bar{m}_{\mathrm{e}}-\bar{m}_{0}\right)\left(1-\mathrm{e}^{4 \bar{m}_{0} x_{0}}\right), \\
F & =\left(\chi \bar{m}_{0}-\bar{m}_{\mathrm{e}}\right)\left(m_{\mathrm{e}}-m_{0}\right), \\
G & =-\left(\chi \bar{m}_{0}+\bar{m}_{\mathrm{e}}\right)\left(\bar{m}_{\mathrm{e}}+\bar{m}_{0}\right) \mathrm{e}^{4 \bar{m}_{0} x_{0}}, \\
H & =-2 \bar{m}_{\mathrm{e}} x_{0}\left(\bar{m}_{\mathrm{e}}+\bar{m}_{0}\right)^{2} \mathrm{e}^{4 \bar{m}_{0} x_{0}},
\end{aligned}
$$

where $\chi=\rho_{\text {extern }} / \rho_{\text {slab }}$, the density contrast ratio. It can be easily seen, by considering the extreme cases of $\bar{\omega}=C_{\mathrm{A} 0} k$ $\left(\bar{m}_{0}=0\right)$ and $\bar{\omega}=C_{\mathrm{Ae}} k_{z}\left(\bar{m}_{\mathrm{e}}=0\right)$, that $\mathfrak{R} \geq 0$. Also, $\bar{m}_{\mathrm{e}} \bar{m}_{0}$ is positive and purely imaginary. Therefore, $\mathfrak{J} \gamma \geq 0$, which corresponds to damping due to wave tunneling. This expansion is only valid in the limit of a thick tunneling region, but in the simulations presented in the previous section, the tunneling region is of equivalent thickness to the slab width. To compare the theory with the simulations, roots to the full dispersion relation are found numerically. These are compared with the results from the simulations in the next section.

In order to understand the mechanism of wave tunneling in our model, we consider the energy budget of the slab. An equation for the energy in the slab, in the absence of driving and dissipation, can be written as

$\frac{\mathrm{d}}{\mathrm{d} t} E=-F$,

where $E$ is the energy in the slab and $F$ is the energy flux through the tunneling region. However, it is also assumed that there is an exponential decay in the energy in time

$\frac{\mathrm{d}}{\mathrm{d} t} E=-\gamma E$,

where $\gamma$ is the decay constant for the energy. This can be equated to give the relation that $\gamma=F / E$. It is reasonable to assume that the energy in a wave is proportional to the square of the amplitude, as in EM theory. Therefore,

$\gamma \propto \frac{F}{A_{0}^{2}}$,

where $A_{0}$ is the amplitude of the waves inside the slab, it is not necessary to specify which of the basic variables is being considered, since the same approach could be applied to any oscillating quantity. If a travelling wave is emitted at the outer boundary, then a single wavelength $\lambda=2 \pi / k$, where $k$ is the magnitude of the wavevector defined as $k=\left(k_{z}^{2}+m_{\mathrm{e}}^{2}\right)^{\frac{1}{2}}$ and equal to $\omega / C_{\mathrm{A} 1}$, is emitted in a time $2 \pi / \omega$. Making the same assumption that the energy contained in a wave is related to the square of the amplitude then the energy flux in a time $2 \pi / \omega$ is $(2 \pi F) / \omega=\int_{0}^{\lambda} A_{\mathrm{e}}^{2} \sin ^{2}(k x) \mathrm{d} x=A_{\mathrm{e}}^{2} \pi / k$, where $A_{\mathrm{e}}$ is the amplitude of the wave in the external region. This simplifies to

$F=A_{\mathrm{e}}^{2} \frac{\omega}{2 k}$.

To complete the analysis, it is necessary to compare the amplitude of the waves inside and outside the slab. This can be done by considering the previous problem (Fig. 15), and explicitly solving for the amplitudes of the waves, but a simpler model yields useful results. The simplest model is to consider a single step function in the Alfvén speed, with the wave travelling from 


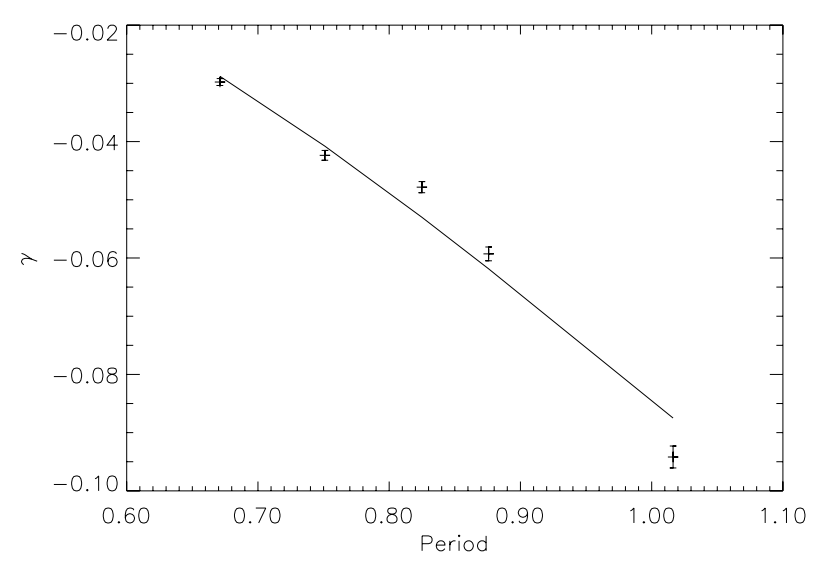

Fig. 16. A fit using the simple model to the obtained decay vs. mode period data for the straight slab.

the tunneling region into the external region, with a given amplitude specified at the beginning of the tunneling region. This is solved using the same methods and approximations as the previous approach. The solution is trivially simple, and yields that the amplitude outside the barrier is

$A_{\mathrm{e}}=C A_{0} \mathrm{e}^{-m_{\mathrm{e}} T}$,

where $T$ is again the width of the tunneling region. The factor $C$ is a constant, $C \leq 1$, which represents the amplitude of the wave at the outer edge of the slab relative to the amplitude of the trapped mode in the slab. The exact value of $C$ is dependant upon the detailed solution of the system, so it is not possible to use this simple model quantitatively. Combining Eqs. (21)-(23) yields the result

$\gamma \propto \mathrm{e}^{-2 m_{\mathrm{e}} T} \frac{\omega}{k}$

The origin of the $\omega / k$ term is purely in the external medium, and since $\omega$ is fixed by the mode, $k$ not in fact a variable for a given $\omega$, and so can be dropped. This immediately yields the expected result that in the limit $T \rightarrow \infty, \gamma \rightarrow 0$. Since $m_{\mathrm{e}}$ is already defined by Eq. (13), and remembering that $k_{z}^{2}=\omega^{2} / C_{\mathrm{ph}}^{2}$ where $C_{\mathrm{ph}}$ is the local phase speed, this can be further rewritten as

$\gamma \propto \omega \mathrm{e}^{-2 \omega\left(\frac{1}{C_{\mathrm{ph}}^{2}}-\frac{1}{C_{A_{1}}^{2}}\right)^{0.5} T}$.

This yields that the decay constant should scale as $\omega \mathrm{e}^{-\omega} \sim$ $\mathrm{e}^{-\frac{1}{P}} P^{-1}$, where $P$ is the wave period.

This prediction can easily be tested by attempting to fit the results obtained from the simulations with a curve with this functional form. The results for the straight slab with this fit are in Fig. 16, and for the original curved slab from Paper I are in Fig. 17. The error values for both fits show an improvement over the straight line fits used in Paper I. Both of these figures is fitted with a function of the form $A / P \mathrm{e}^{-\frac{B}{P}}+C$, where $A$, $B$, and $C$ are constants which are used to specify the fit. The values of the fitting constants are given in the figure captions. In theory, $C$ should be zero, but will have a non zero value due to the presence of finite viscosity.

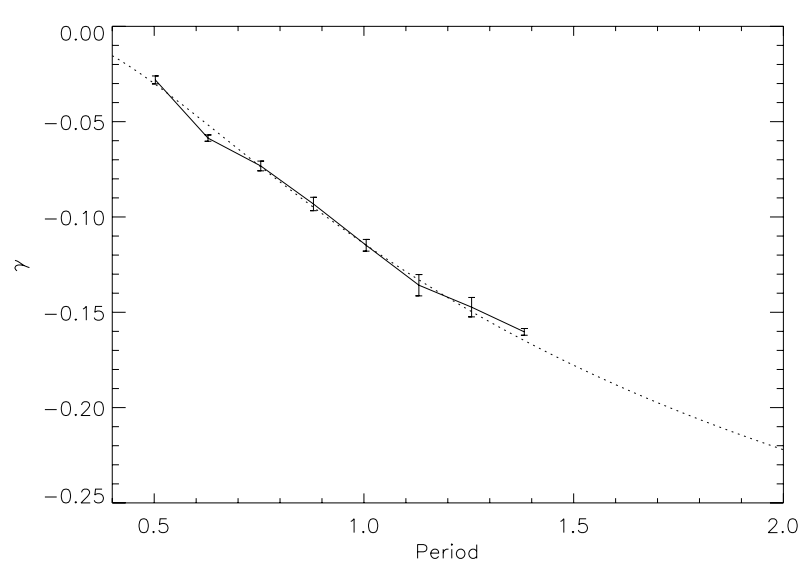

Fig. 17. A fit using the simple model to the obtained decay vs. mode period data from Paper I. The extrapolated decay time for the fundamental mode using this model is twice that obtained in the original paper. The fitting constants are $A=-1.16 \pm 0.01, B=-2.40 \pm 0.01$ and $C=-0.01$.

The extrapolated decay times for the fundamental mode are increased by a factor of 2 compared with the previous straight line extrapolation. An exponential fit to the decay rate vs. tunneling thickness is also the best fit out of linear, quadratic, and exponential fits, suggesting that this prediction of this model is correct as well. The derivation of this model includes too many simplifications for any quantitative results to be taken from it, and comparison with simulations shows that this is the case. The more complete derivation however can be used to give more quantitative results. Although the magnetic slab model is much simpler than that used in the simulations, it is hoped that the impact of the details of the slab model are not significant.

\section{Discussion}

We have extended and examined the work of Brady \& Arber (2005), considering in more detail the effect of wave tunneling on fast kink modes in coronal loops. To this end, a straight slab model incorporating a variable tunneling region was designed and both compared to the previous results relating decay time to mode period. The effect of barrier thickness on the decay time of the modes has been investigated. Two analytical models were also constructed. The first model was a modification of the Roberts (1981) model for wave modes of a magnetic slab to incorporate a tunneling region. This model yields an analytical dispersion relation for linear waves in a straight slab with a finite thickness tunneling region, but this relation is transcendental and requires roots to be found numerically. To allow a qualitative understanding of the dependence of the model on the parameters, a simpler model was constructed by considering the effect of the tunneling region alone. This model gives no useful quantitative data, but by comparison with the numerical simulations accurately reproduces the functional dependency of the decay time on both the thickness of the tunneling region and the period.

In Paper I, it was simply inferred that the cause of the observed decay was the leakage of waves by tunneling loss, and no attempt was made to rigorously prove that this was the case. 
Here, simulations of a magnetic slab that in the absence of a tunneling region would act as a waveguide, shows that the introduction of a tunneling region introduces a finite leakage rate, and finite decay time. This decay is found to have the same functional form as that for the simulations of curved slabs, but has a significantly longer decay time for the same standing harmonic. This effect can be explained by a simple geometric correction to the model. It was stated earlier that a mode trapped in the slab can be represented by a line of constant $\omega / k$, which is what leads to the definition of the tunneling thickness as the distance from the slab centre until the Alfvén speed is again lower than that in the slab centre. For a straight slab, this is correct, but more generally the tunneling thickness is the distance along the line $\omega / k_{\mathrm{z}}$ representing the mode from the point where the mode ceases to be propagating, until the point where it becomes propagating again. For a curved slab $k$ must decrease for increasing radius, otherwise the mode structure would not be preserved for all radii. This leads to a relationship of $\omega / k \propto r$, where $k=m / r$ is associated with the longitudinal wavenumber. The value of $\omega / k$ in the slab itself can be obtained from the simulations, so the constant of proportionality can be obtained. This allows a new tunneling thickness to be calculated for the curved slabs, which is always lower than the tunneling thickness for the equivalent straight slab. Combined with the theory, which gives $\gamma \propto \mathrm{e}^{-T}$ where $T$ is the thickness of the tunneling region, this allows a correction factor to be applied for a given mode number, which is

$\gamma_{\text {straight }} \propto \gamma_{\text {curved }} \mathrm{e}^{-2 \omega\left(\frac{1}{c_{\mathrm{A} 0}^{2}}-\frac{1}{C_{\mathrm{ph}}^{2}}\right)^{\frac{1}{2}} \Delta T}$,

where $\Delta T$ is the difference in the tunneling widths between the curved and straight slabs, and all other symbols have their previously given meanings. The value of $\Delta T$ can be calculated by comparing the thickness of the tunneling region for a simulation of $n=2$, and $n=4$. This is because in the case $n=2$ the Alfvén speed profile is exactly the same in the straight case as it was in the curved case, but for $n=4$, it is equivalent to having the same Alfvén speed profile as for the curved slab divided by $r$. It can therefore been seen as equivalent to dividing the $y$-axis in Fig. 2 by $r$. Therefore, since the lines representing modes for a curved slab in Fig. 2 are lines of the form $A r$, where $A$ is a constant, dividing the $y$-axis by $r$ makes these into horizontal lines. For a straight slab, these lines for a given mode are already horizontal lines, and so the two systems now have an equivalent tunneling thickness. This can be obtained trivially from the definition of the Alfvén speed profile (Eq. (9)) and the fact that $\omega / k=C_{\mathrm{A} 0} \propto r / r_{0}$. The value of $\Delta T$ can then be obtained numerically. Comparing values from the straight slab and curved slab results using this correction formula yields values which agree to within 5\% at worst (shown in Fig. 18). This strongly suggests that the same mechanism is responsible for the leakage in both the straight and the curved slab geometries, and that the mechanism in both cases is consistent with a simple model of wave tunneling. The results for the straight slab can be confirmed to be wave tunneling by comparing the results of the simulation with the results from the more complete analytical model for the dispersion relation for waves in

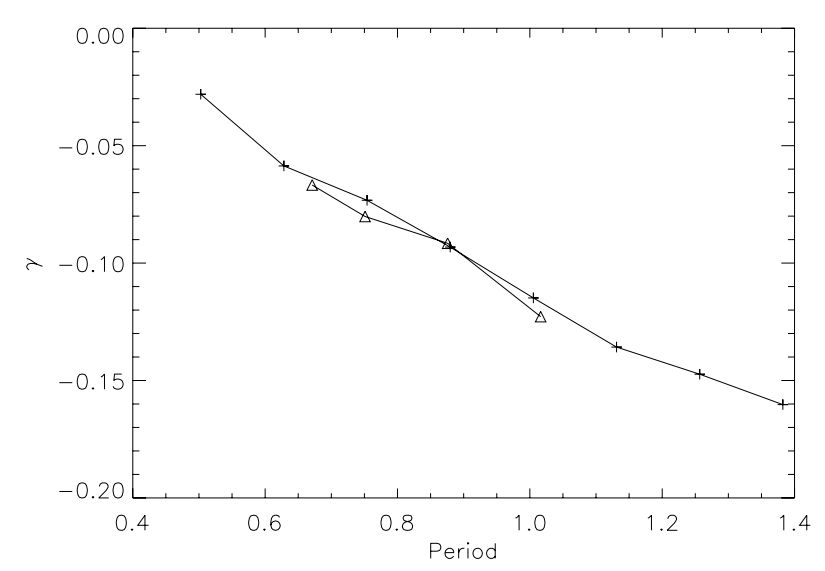

Fig. 18. The results from Paper I are plotted with star symbols, and the results from Fig. 16 are plotted after correction with Eq. (26) with triangle symbols.

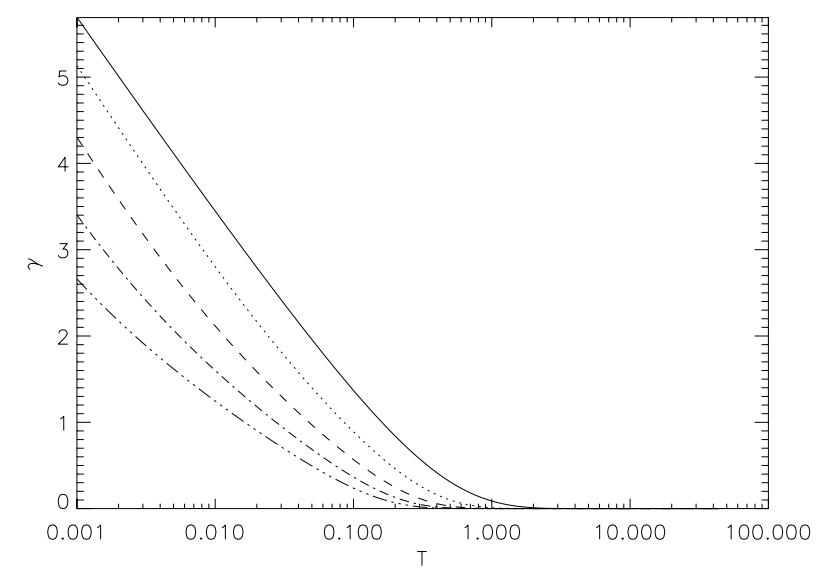

Fig. 19. The value of $\gamma$ obtained from the more detailed theoretical model presented earlier against $T$. The different lines represent different values of $k_{z}$. The solid line is for the first harmonic, the dotted line the second harmonic, the dashed line the third harmonic, the dotdashed line the fourth harmonic, and the triple-dot-dashed line the fifth harmonic.

a slab with a tunneling region. As with the results for a simple magnetic slab, the analytical model gives several branches of solution, but only the kink mode branch will be considered here. The dispersion relation obtained is transcendental, but as already stated an approximate solution can be obtained when the decay is weak. The following results use a combination of the analytical approximation and a numerical solution to the dispersion relation, with the switchover between the two occurring when the difference between the two solutions is one part in $10^{4}$. The numerical root finding algorithm used here finds the full complex values of the phase speed of the waves in the slab as a function of $k_{z}$, the wavenumber in the tunneling region, and $T$, the thickness of the tunneling region. The real part of the phase speed corresponds to the oscillatory part of the solution for the wave in the slab, and so is compared with the period from the simulations. The imaginary part corresponds to the damping, and so is compared with the decay time from the simulations. 


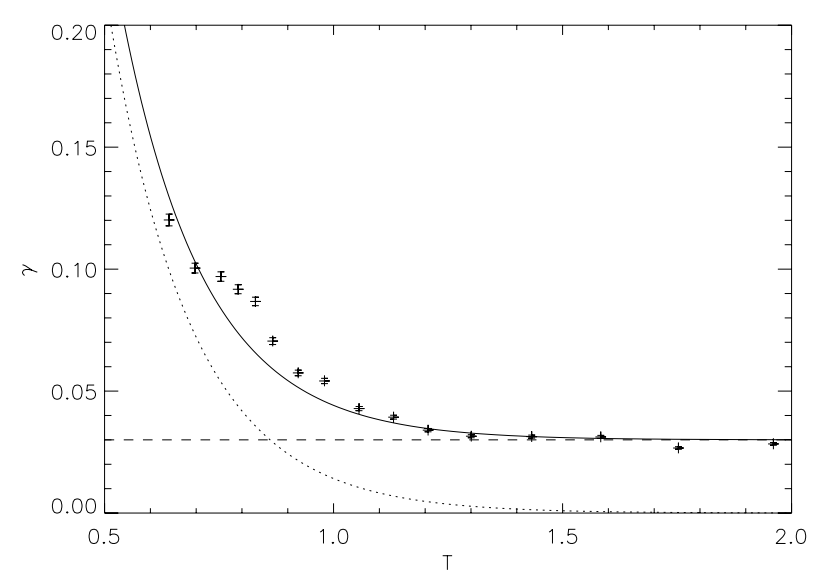

Fig. 20. The values of decay constant $\gamma$ vs. tunneling thickness $T$ are from Fig. 12, and are compared to the equivalent values of decay constant from the more complete theory. The density contrast ratio is 10 , and the ratio of the slab length to the width is 0.2 . The plot has been modified to correct for the presence of finite decay due to viscosity. Note that there are no fitted parameters in the theoretical model. The dotted line represents the line for $\gamma$ uncorrected for viscous damping, and the dashed line represents the calculated decay value due to viscous damping.

A series of plots of the theoretical values for the decay constant is given in Fig. 19, for a variety of values of $k_{z}$. By calculating the value of $k_{z}$ for a simulation, the value of the decay constant vs. tunneling thickness can be compared with that from the theory (see Fig. 20).

The results agree to within error with the values obtained from theoretical calculations. Given that the Alfvén speed profile used in the numerical simulations is significantly more structured than that of the analytical model, this shows that the tunneling effect is primarily sensitive to the thickness of the tunneling region, not the details of the Alfvén speed profile. This is confirmed by comparing the predicted and obtained periods of the fast mode in the slab, which also agree to within the errors associated with the fitting of the curves produced by the numerical simulations with those observed from the simulations. Results for other periods also show similarly good matches with theory.

The results in this paper rely upon the derivation of a model which is equivalent to the curved slab model in Brady \& Arber (2005), but without the added complication of curvature. The model chosen in this paper is a simple modification of a straight slab model to allow the introduction of a finite thickness tunneling region, and has the consequence of changing the density profile so that the density increases outwards from the slab. In the zero plasma- $\beta$ limit, the only relevant parameter is the Alfvén speed profile, with the density not being directly involved in the governing equations. Therefore, it would be expected that even in the finite plasma- $\beta$ case of the simulations the density profile will only weakly affect the answers given by the simulation.

Unlike the previous paper, the aim of this paper is not to compare directly with observations, but is to confirm the interpretation of the previous results. It achieves this by changing the geometry of the system so that the flux slab is straight, and uses a simple analytical model to change the curvature into a geometric correction. The good agreement between simulations and theory suggests that the interpretation of the results in the previous paper as leakage by wave tunneling is correct.

This then begs the further question of the role that the tunneling effect has on the leakage of waves from coronal loops. Neither this paper nor the preceding one can fully describe the effect of wave tunneling on a realistic coronal loop. A better understanding of the role of this mechanism requires further simulations to consider more realistic 3D loops with the inclusion of gravity.

\section{References}

Arber, T. D., Longbottom, A. W., Gerrard, C. L., \& Milne, A. M. 2001, JCP, 170, 151

Aschwanden, M. J., Fletcher, L., Schrijver, C. J., \& Alexander, D. 1999, ApJ, 620, 880

Brady, C. S., \& Arber, T. D. 2005, A\&A, 438, 733

Van Doorsselaere, T., Debosscher, A., Andries, J., \& Poedts, S. 2004, A\&A, 424, 1065

Nakariakov, V. M., Ofman, L., DeLuca, E. E., Roberts, B., \& Davila, J. M. 1999, Science, 285, 862

Ofman, L., \& Aschwanden, M. J. 2002, ApJ, 576, 2, L153

Roberts, B. 1981, Sol. Phys., 69, 39

Roberts, B. 2000, Sol. Phys., 193, 139

Roberts, B., Edwin, P. M., \& Benz, A. O. 1983, Nature, 305, 688

Roberts, B., Edwin, P. M., \& Benz, A. O. 1984, ApJ, 279, 857

Ruderman, M. S., \& Roberts, B. 2002, ApJ, 577, 475

Selwa, M., Murawski, K., Solanki, S. K., Wang, T. J., \& Tóth, G. 2005, A\&A, 440, 385

Wang, T. J., \& Solanki, S. K. 2004, A\&A, 421, L33

Verwichte, E., Foullon, C., \& Nakariakov, V. M. 2006, A\&A, 446, 1139 